Société d'histoire de la révolution de 1848 et des

révolutions du XIXe siècle

$58 \mid 2019$

Histoire et archéologie : que faire du XIXe siècle ?

\title{
La tombe de Jeanne (1877 - ). Histoire et archéologie
}

Jeanne's Grace (1877- ...) History and archeology

Das Grab von Jeanne (1788-...). Geschichte und Archäologie

\section{Bruno Bertherat}

\section{(2) OpenEdition}

Journals

Édition électronique

URL : https://journals.openedition.org/rh19/6426

DOI : $10.4000 /$ rh 19.6426

ISSN : $1777-5329$

Éditeur

La Société de 1848

Édition imprimée

Date de publication : 1 août 2019

Pagination : 21-40

ISSN : 1265-1354

Référence électronique

Bruno Bertherat, «La tombe de Jeanne (1877 - ). Histoire et archéologie », Revue d'histoire du XIXe siècle [En ligne], 58 | 2019, mis en ligne le 01 janvier 2022, consulté le 07 juillet 2022. URL : http:// journals.openedition.org/rh19/6426; DOI : https://doi.org/10.4000/rh19.6426 


\section{BRUNO BERTHERAT}

\section{La tombe de Jeanne (1877 - ). Histoire et archéologie}

La tombe de Jeanne est encore visible aujourd'hui au cimetière communal de Bagneux (Hauts-de-Seine, ancien département de la Seine), même si le nom qu'elle porte est quasi effacé depuis longtemps. Elle est devenue vestige. Spécialistes du passé et bien souvent des choses mortes, les historiens et les archéologues connaissent les tombes. Alors que l'archéologie du funéraire récent se développe depuis quelques années ${ }^{1}$, l'article présenté ici tente de montrer l'importance de son utilisation dans la réflexion historique à partir de l'étude en cours d'une sépulture. Celle-ci contient le cadavre de Jeanne Moyaux, petite victime de quatre ans d'un célèbre fait-divers criminel survenu en 1877. Jetée vivante dans un puits par son père, Victor Moyaux, à Bagneux où habitait son grand-père maternel et où elle avait un peu vécu, l'enfant mourut à l'hôpital Cochin à Paris, puis fut exposée à la Morgue sur l'île de la Cité pour y être identifiée avant d'être inhumée dans le terrain commun du cimetière de Bagneux après de grandes funérailles. Son corps fut peu après transféré dans une concession perpétuelle et pourvu d'un tombeau. La nature du crime, ses péripéties ainsi que son éclat public et médiatique ${ }^{2}$ ont rejailli sur la tombe et lui ont assuré une sorte de pérennité.

Mais l'anecdotique est ici au service d'un exercice de méthode. En nous invitant à décrypter le "langage muet des choses $^{3}$ ", équivalent en richesse à celui des mots, et à les faire dialoguer dans une approche globale, cette tombe d'enfant atypique permet d'appréhender le système funéraire au centre duquel elle s'inscrit. Le culte des morts, tel qu'il se dessine dans le courant du XIX e siècle à la suite d'une "transition funéraire " amorcée à l'époque des

1. Bruno Bertherat, "L'historien, l'archéologue et la mort (France, époque contemporaine)", in Nicolas Weydert, Stéfan Tzortzis, Anne Richier, Laëtitia Lantéri et Hervé Guy (dir.), Rencontre autour de nos aïeux. La mort de plus en plus proche. Actes de la $8^{c}$ Rencontre du Groupe d'anthropologie et d'archéologie funéraire, 25-27 mai 2016, Faculté de la Timone, Marseille, Reugny, Gaaf, 2019, p. 61-70.

2. Sur les faits divers, on renvoie principalement à Dominique Kalifa et Anne-Claude AmbroiseRendu.

3. Alain Schnapp, «Introduction», in Alain Schnapp (dir.), L'Archéologie aujourd'hui, Paris, Hachette, 1980, p. 21.

4. Régis Bertrand, Mort et mémoire. Provence, XVIII'-XX' siècles. Une approche d'historien, Marseille, La Thune, 2011, p. 5-18. 
Lumières et balisée par le décret du 12 juin 1804 (23 prairial an XII), avant de connaître un relatif déclin aujourd'hui, donne en effet à la tombe, dont le cimetière devient en quelque sorte l'écrin, une importance inédite : le souvenir, familial le plus souvent, a désormais son temple 5 . Il importe donc de saisir cette tombe dans toutes ses dimensions : l'espace qu'elle occupe, visible et invisible, et les deux temporalités qui déterminent son existence, le temps de sa création et celui de ses usages jusqu'à ce jour où nous écrivons ces lignes. Après avoir présenté le panorama des sources historiques et archéologiques, avec leur complémentarité et leurs limites, l'historien tentera de décrire le temps figé du souvenir, matérialisé par la tombe de Jeanne, puis l'usure du temps qu'elle ne peut empêcher.

\section{DES «CATACOMBES MANUSCRITES " AUX «ARCHIVES DU SOL ${ }^{6}$ »}

L'historien comme l'archéologue pratiquent un travail d'exhumation, mais la poussière qu'ils soulèvent n'est pas de même nature. Les mots et les archives sont la source principale du travail historique; les choses et les strates du sol celle du travail archéologique. Cependant, la porosité même du concept de source invite à nuancer ces oppositions.

Dans son approche de la tombe, l'historien bénéficie de multiples sources qui sont essentiellement celles des cimetières ${ }^{7}$. Celles de Bagneux se trouvent d'abord aux archives municipales et à la conservation du cimetière, avenue de Garlande (anciennement avenue du Cimetière). Elles permettent de comprendre l'histoire du lieu à l'échelle du territoire de la commune, qui est à l'époque de la mort de Jeanne un gros village d'environ 1500 habitants ${ }^{8}$. C'est dans un cimetière entouré de champs conforme aux normes du décret de prairial qu'est inhumée Jeanne, qui a remplacé dix ans auparavant l'ancien

5. Philippe Ariès, L'Homme devant la mort, Paris, Le Seuil, 1977; Michel Vovelle, La Mort et l'Occident de 1300 à nos jours, Paris, Gallimard, 1983. Voir aussi Arnold Van Gennep, Le Folklore français, vol. 1, Du berceau à la tombe. Cycles de carnaval-carême et de Pâques, Paris, Robert Laffont, 1998 [1943, 1946, 1948], p. 557-702; Thomas Kselman, Death and the Afterlife in Modern France, Princeton, Princeton University Press, 1993; Régis Bertrand, Les Provençaux et leurs morts. Recherches sur les pratiques funéraires, les lieux de sépultures et le culte du souvenir des morts dans le Sud-Est de la France depuis la fin du XVIT siècle, thèse d'histoire, Université Paris 1 Panthéon-Sorbonne, 1994; Madeleine Lassère, Villes et cimetières en France de l'Ancien Régime à nos jours. Le territoire des morts, Paris, L’Harmattan, 1997; Régis Bertrand, Mort et mémoire..., op. cit.; Bruno Bertherat (dir.), Les Sources du funéraire en France à l'époque contemporaine, Avignon, Éditions universitaires d'Avignon, 2015; Régis Bertrand et Anne Carol (dir.), Aux origines des cimetières contemporains. Les réformes funéraires de l'Europe occidentale, XVIII-XIX siècle, Aix-en-Provence, PUP, 2016; Régis Bertrand et Guénola Groud (dir.), Cimetières et tombeaux. Patrimoine funéraire français, Paris, Éditions du patrimoine, 2016.

6. La première expression est de Jules Michelet, Histoire de France, Paris, L. Hachette, 18331841 , t. 2, p. 701-702. Nous ignorons l'origine de la seconde, fréquemment utilisée quand il s'agit d'archéologie.

7. Régis Bertrand, «Les sources d'une monographie de cimetière», in Bruno Bertherat (dir.), Les Sources..., op. cit., p. 49-64.

8. État des communes à la fin du XIXe siècle. Bagneux. Notice historique et renseignements administratifs, Montévrain, Imprimerie typographique de l'école d'Alembert, 1901, p. 36-37. 
cimetière situé sur l'actuelle place Dampierre, jugé trop proche des habitations, et près duquel s'installe presque une décennie plus tard l'immense cimetière parisien de Bagneux (fig. 1). L'organisation du cimetière nous est connue par un règlement de 1868 , mais il n'existe pas de plan précis avant son agrandissement dans les années $1930^{\circ}$. Intéressants pour comprendre la vie du cimetière, les procès-verbaux des séances du conseil municipal restent muets sur la tombe de Jeanne. Celle-ci apparaît dans le registre des inhumations et dans une fiche individuelle, ainsi que dans divers dossiers concernant les concessions, signe de l'attention portée par la municipalité à la gestion du cimetière, même s'il y a un manque de données sur le suivi de la tombe provoqué peut-être par la numérisation des archives de la conservation $^{10}$. En revanche, les archives de l'état civil de Jeanne sont localisées à Paris, puisqu'elle est née et morte dans cette ville où habitaient ses parents à l'époque ${ }^{11}$. Ces archives se limitent toutefois à des données factuelles. Aussi le dossier de la souscription pour l'érection d'un monument funéraire est-il exceptionnel, car il nous fait rentrer dans une histoire sensible de la tombe, malgré le temps écoulé12.

D'autres sources permettent de multiplier les éclairages sur la tombe de Jeanne. Il y a les sources normatives au sens large, soit juridiques (les lois et règlements sur les cimetières en général), soit esthétiques avec en particulier les albums de tombeaux, et les sources descriptives et iconographiques sur le cimetière et la commune de Bagneux. À ce stade de notre enquête, nous ne disposons pas de photographies ni de descriptions du cimetière dans le passé, ce qui pourrait s'expliquer en partie par sa faible notoriété. Parcellaires et focalisées sur le crime et sa résolution, ainsi que sur le criminel, les sources judiciaires et médiatiques sur la mort de Jeanne ne s'intéressent guère à la tombe, même si les funérailles, (peu ou mal) encadrées par les forces de l'ordre, bénéficient d'une large couverture ${ }^{13}$. Les archives religieuses sont également décevantes, alors que la petite fille, qui était baptisée, a été semblet-il accueillie un temps par les sœurs de Saint-Vincent-de-Paul dans leur établissement de Bagneux et a eu des funérailles religieuses ${ }^{14}$. Même constat

9. Archives communales de Bagneux (ACB), W8 (non classé), Dossier Ancien tarif et nouveau.

10. Conservation du cimetière municipal de Bagneux, ACB, $271 \mathrm{~W} 23$ et 35.

11. Jeanne a eu deux actes de décès, ce qui est anormal : le premier, incomplet, dressé dans le $\mathrm{XIV}^{\mathrm{e}}$ arrondissement où se trouve l'hôpital Cochin; le second dans le IV $\mathrm{V}^{\mathrm{e}}$ arrondissement où se trouve la Morgue (Archives de Paris, V4E 4434, n 307,5 février 1877 et V4E 2897, ${ }^{\circ}$ 650, 10 mars 1877).

12. ACB, W3 (non classé), Dossier Souscriptions diverses, 1821-1881.

13. Les Archives de Paris n'ont pas conservé le dossier d'instruction qui aurait pu apporter des précisions sur le contexte local. Les Archives de la Préfecture de police (APP) possèdent un dossier intéressant sur l'affaire (BA81). Quant à la presse, elle est parisienne. Ce n'est qu'à la fin du siècle qu'apparaît une presse locale en région parisienne, en tous cas dans la zone de Bagneux. Nous n'avons pas étudié ici l'écho médiatique de l'affaire dans le reste de la France, voire à l'étranger.

14. La paroisse conserve le registre qui mentionne les funérailles de Jeanne. Les archives du diocèse de Paris, celles, plus récentes, du diocèse de Nanterre et celles du gouvernement général de la Compagnie des Filles de la Charité de Saint-Vincent-de-Paul (140 rue du Bac à Paris) ne conservent aucune archive relative à la petite fille. Fondé en 1860 et supprimé en 1903, l'établissement de Bagneux était situé 20 rue d'Arcueil. 


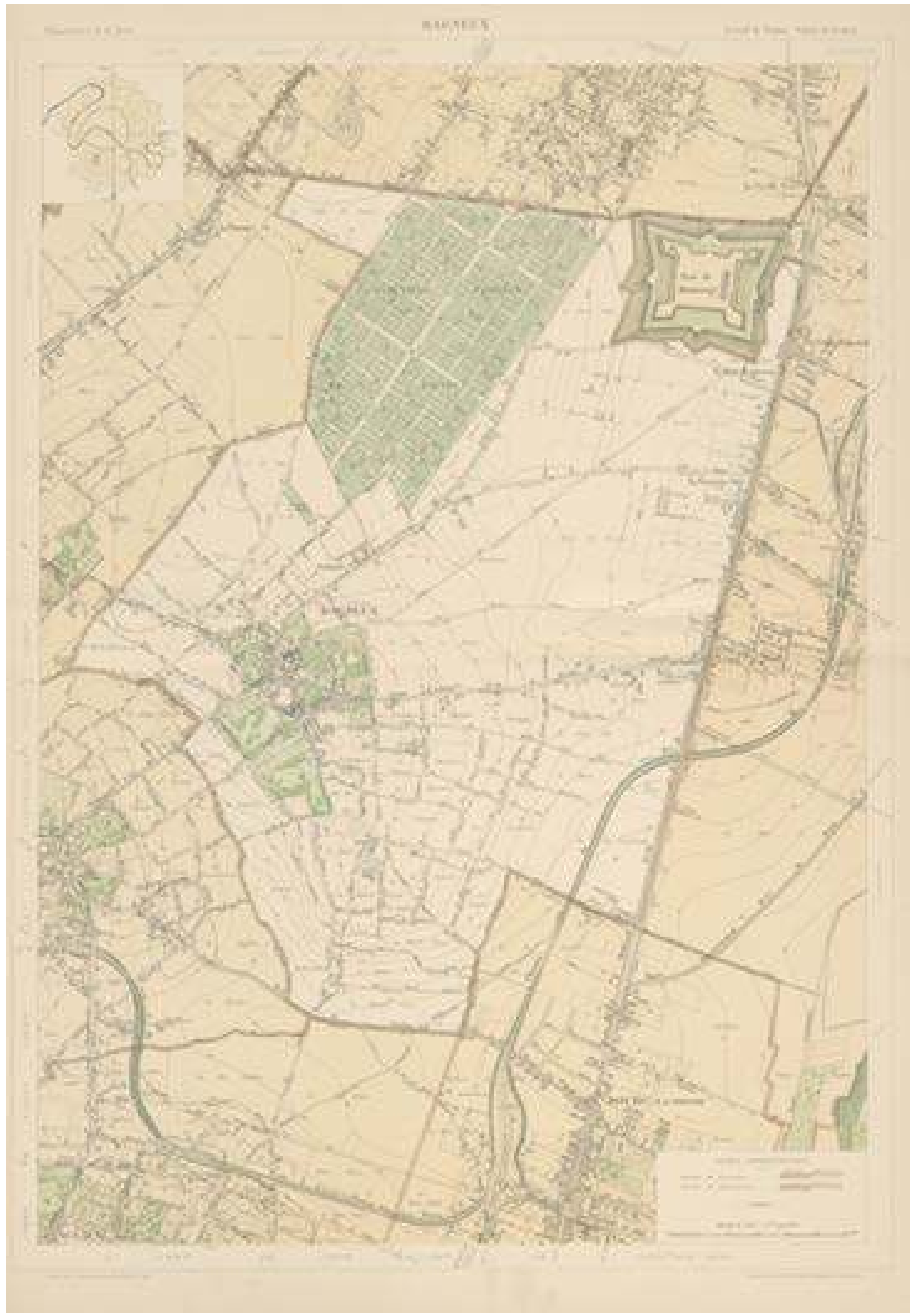

Fig. 1 : Plan de Bagneux, 1894-1900 (Archives départementales des Hauts-de-Seine, 5FI/Bag 3). Le cimetière parisien de Bagneux est ouvert en 1886.

Crédits photographiques : Archives départementales des Hauts-de-Seine. 


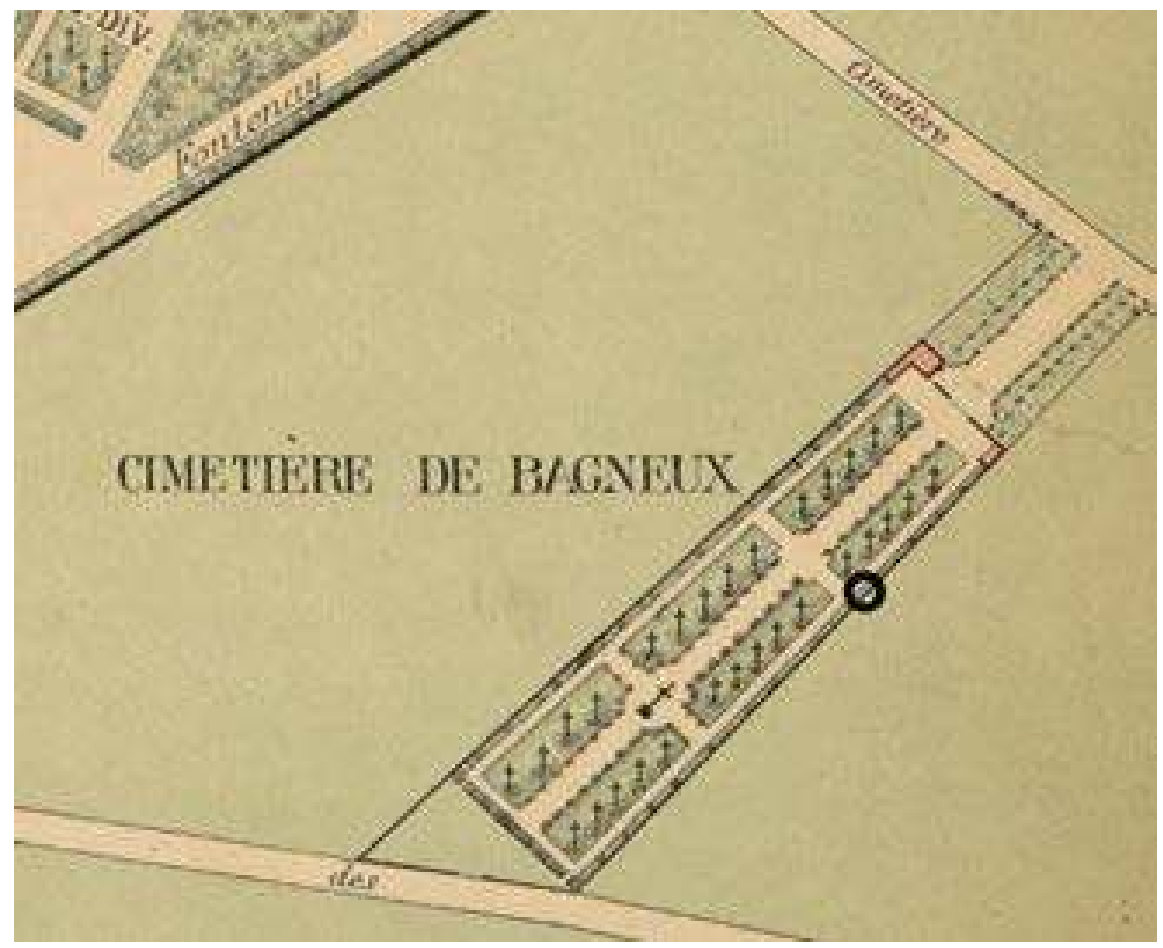

Fig. 2 : Le cimetière municipal de Bagneux, 1905 (Bibliothèque nationale de France, Le Cimetière parisien de Bagneux, 1905, détail). ๑ Gallica.

concernant les archives privées, comme celles de l'entreprise de maçonnerie François Radigon qui a construit le tombeau de Jeanne ${ }^{15}$. Il y a une exception : la collection de René Rousseau (1906-2007), fondateur et premier président de l'association Les Amis de Bagneux, qui comporte des pièces sur l'affaire, dont les plus anciennes photos de la tombe conservées qui ne datent que de la fin du $\mathrm{Xx}^{\mathrm{e}}$ siècle, mais permettent de faire des comparaisons avec les nôtres ${ }^{16}$. Quant aux sources orales, elles sont a priori inexistantes : la tombe de Jeanne nous renvoie à un contemporain désormais ancien et les échos de ses usages semblent définitivement perdus. L'aspect originel de la tombe et son évolution nous échappent donc presque totalement.

L'historien est habitué à la disparité des sources. Dans cette enquête, la difficulté réside dans l'écart entre l'éclat médiatique du crime et la discrétion

15. Annuaire-almanach du commerce et de l'industrie, Paris, Firmin Didot et Cie, 1877, p. 2062. La société existe encore aujourd'hui quasiment sous le même nom (Radigon-Bioret), mais elle a changé de propriétaire. Il n'y a plus aucune archive de l'époque de Jeanne. Le mémoire de frais pour son tombeau figure néanmoins dans le dossier de souscription.

16. Musée du domaine départemental de Sceaux - Centre de documentation, Collection René Rousseau, 778 à 789. Le fonds a été versé en 1998-1999. La date des photographies n’est pas connue. 
du retour à la norme funéraire. Avec l'archéologie, l’approche prend une autre dimension.

Commencée avec les «sépultures de crises» et les cimetières de l'époque coloniale dans les années $1990^{17}$, l'archéologie funéraire des temps contemporains s'intéresse depuis quelques années aux cimetières métropolitains désaffectés, principalement dans le cadre d'opérations préventives, comme à Antibes, Canet-en-Roussillon, Nancy ou Marseille ${ }^{18}$. En dehors des sépultures de crise (on pense à la Première Guerre mondiale), le cimetière le plus récent ayant été fouillé en France est celui du quartier des Crottes à Marseille, fermé en 1905 et désaffecté en 1926. Il nous servira de référence ${ }^{19}$. Parmi les tombes mises au jour, celle d'un petit garçon entre 5 et 9 ans inhumé sans doute dans les années 1860, qui semble faire écho, à l'autre bout de la France, à la tombe de Jeanne ${ }^{20}$. La fouille manuelle permet d'accéder à la part invisible de la culture matérielle de la mort. Dans le cas de la tombe de Jeanne, elle pourrait donner de précieux renseignements sur ce que ne disent habituellement pas les sources historiques, à savoir le mode d'enfouissement, le type de cercueil, ses accessoires extérieurs et intérieurs, l'état et la disposition du corps et les objets l'accompagnant ${ }^{21}$. Les données apportées par l'anthropologie biologique seraient moins intéressantes, puisque l'identité et les causes de la mort sont connues par les sources historiques (on reviendra plus loin sur l'état de son corps au moment de l'inhumation). Cependant, peu de cimetières de cette période ont été fouillés à ce jour, limitant l'ampleur des comparaisons.

Les cimetières restent en effet difficiles d'accès en raison de leur caractère récent. Ceux encore en activité échappent même à l'archéologue. Tel est le cas du cimetière de Bagneux. La fouille de la tombe du soldat Chassaigne (fusillé pendant la Première Guerre mondiale) menée par Guy Flucher au cimetière communal d'Hartennes dans l'Aisne constitue une exception, mais

17. Les sépultures de crises sont liées à des mortalités d'une ampleur exceptionnelle (épidémies et guerres par exemple). Voir par exemple Frédéric Adam, Alain-Fournier et ses compagnons d'arme. Une archéologie de la Grande Guerre, Metz, Éditions Serpenoise, 2006 (sur une fouille pionnière, qui a eu lieu en 1991); André Delpuech et Jean-Paul Jacob (dir.), Archéologie de l'esclavage colonial, Paris, La Découverte, Inrap, 2014, p. 317-388.

18. Il faudrait ajouter à cette liste les fouilles menées sur des cimetières dont l'activité s'est étendue sur une très longue période.

19. Dans le $\mathrm{Xv}^{\mathrm{e}}$ arrondissement. Voir Anne Richier et Nicolas Weydert (dir.), Ancien cimetière des Crottes. Rapport d’opération. Inrap Méditerranée, avril 2016, 6 vol. Les références des rapports des autres fouilles citées (1999, 2008 et 2012) sont disponibles dans les services régionaux d'archéologie concernés et éventuellement sur Dolia, le catalogue des fonds documentaires de l'Inrap.

20. Id., vol. 1, p. 452-454.

21. Les fournitures se retrouvent dans les archives des entreprises de pompes funèbres, rarement conservées pour des périodes aussi anciennes. Dans le cas de constatations ou d'exhumations judiciaires, les sources indiquent parfois ce que l'on trouve dans le cercueil : Sandra Menenteau, «Du funéraire dans le judiciaire. Les procédures criminelles et correctionnelles du département de la Vienne au XIX ${ }^{\mathrm{e}}$ siècle», in Bruno Bertherat (dir.), Les Sources..., op. cit., p. 217-239. Parmi les autres sources possibles, la presse, les romans, les récits familiaux, la correspondance privée, les photographies mortuaires. 
la sépulture allait être supprimée ${ }^{22}$. Ce qui n'est pas le cas de la concession perpétuelle dans laquelle repose Jeanne. Une fouille programmée dans un but scientifique serait possible, à condition qu'elle s'insère dans un programme national de recherche adossé à des laboratoires, ce qui n'est pas le cas ici. Difficile à justifier pour la tombe de Jeanne, cette fouille est-elle même éthiquement souhaitable? Ne remet-elle pas en cause la finalité même de la concession perpétuelle et les volontés de celles et ceux qui l'ont choisie pour elle? Par ailleurs, les méthodes de prospection non intrusives, comme la photographie aérienne, la technologie du scanneur laser aéroporté ou $L I D A R^{23}$ et même la prospection magnétique ne permettent pas aujourd'hui d'accéder à l'intimité de la tombe enfouie et ne sauraient a priori remplacer l'intervention physique : l'œil de l'archéologue n'est-il pas le prolongement de sa main? La sépulture de Jeanne est donc encore protégée par la loi. Toute intervention remettant en cause son intégrité, car la fouille détruit autant qu'elle découvre, s'apparenterait à une violation ou à une profanation de sépulture, voire à une atteinte à l'intégrité du cadavre ${ }^{24}$.

On peut alors se demander si la difficulté de la fouille manuelle, voire son impossibilité concernant la tombe de Jeanne, rend le questionnement archéologique pertinent. Pour essayer de résoudre la contradiction, il faut remonter à la surface.

C'est en effet à la surface des choses, à hauteur de tombeau, que le dialogue entre l'historien et l'archéologue peut se nouer, car leur territoire se chevauche. Le premier s'occupe à sa manière de «faire parler les choses muettes ${ }^{25}$ » et pratique de longue date l'enquête de terrain, à l'exemple de l'anthropologue et du sociologue. Michel Vovelle et Régis Bertrand ont ainsi parcouru les cimetières provençaux pour mieux en décrire le paysage et son évolution ${ }^{26}$. L'archéologue, quant à lui, sait tirer profit de l'étude de la surface quand les circonstances l'imposent. C'est ainsi que l'opération sur le cimetière juif de l'Isle-sur-la-Sorgue (Vaucluse), abandonné mais non désaffecté, menée en 2013 par François Guyonnet a permis de dégager des sépultures de leur gangue végétale et de numériser l'ensemble de l'espace, lui redonnant toute sa cohérence ${ }^{27}$. Il faut aussi évoquer l'intérêt développé il y a plusieurs

22. Guy Flucher et Denis Rolland, «Le sujet Chassaigne : fusillé pour l'exemple ou droit commun", La Lettre du Chemin des Dames, 31, 2014, p. 9-11.

23. Light detection and ranging.

24. Articles 225-17 et 18 du Code pénal. La violation de sépulture figurait déjà dans l'article 360 du Code pénal de 1810. Voir Amandine Malivin, Voluptés macabres. La nécrophilie en France au XIX siècle, Thèse d'histoire, Université Paris 7, 2012.

25. Lucien Febvre, Combats pour l'histoire, Paris, Armand Colin, 1953, p. 428.

26. Michel Vovelle et Régis Bertrand (dir.), La Ville des morts. Essai sur limaginaire urbain contemporain d'après les cimetières provençaux, Paris, CNRS, 1983.

27. Tessa Boehm, François Guyonnet, David Lavergne, Jean Marx et Marc Sylvain, "La redécouverte du cimetière juif de L'Isle-sur-la-Sorgue (Vaucluse). Une première approche historique et archéologique au service d'un projet de mise en valeur», in Bruno Bertherat (dir.), Les Sources..., op. cit., p. 189-210. 
décennies déjà par Philippe Bruneau et Pierre-Yves Balut ${ }^{28}$, au sein du Centre d'archéologie générale de l'université Paris IV et de sa revue RAMAGE qu'ils avaient créés, pour une approche fondée non plus seulement sur la fouille, mais aussi sur l'observation et sur la documentation des usages. Dans cette approche, les enquêtes de terrain sont combinées à une volonté de théorisation $^{29}$. On retrouve cette attention portée à l'objet dans le travail des historiens de l'art et des conservateurs du patrimoine ${ }^{30}$. Il existe même depuis peu des recensions ou des collectes des traces des pratiques funéraires, notamment sur les tombeaux, mais elles sont plutôt réservées aux événements et aux personnalités exceptionnels ${ }^{31}$.

Ces différentes approches complètent les précédentes; ensemble, elles donnent de la profondeur au temps et aux choses. La complémentarité entre l'historien et l'archéologue des temps contemporains est de plus en plus importante, comme en témoignent le caractère parfois pluridisciplinaire des rapports de fouilles ainsi que la large place accordée aux sources historiques, et les publications historiennes qui intègrent désormais l'apport de la démarche archéologique. Il ne faut pas négliger non plus les témoignages des professionnels des pompes funèbres et du cimetière, car l'étude des pratiques du présent permet de mieux comprendre celles du passé. Conservateurs et fossoyeurs savent à quoi ressemblent les entrailles du cimetière et comment elles évoluent avec les corps qu'ils y déposent et qu'ils en retirent pour les déplacer : la proximité avec le travail de l'archéologue est réelle (les gestes à cet égard nécessiteraient une comparaison fine), même si la temporalité et les finalités ne sont pas les mêmes. Différence essentielle, l'archéologue n'intervient qu'à la fin du processus : il ne met pas en terre. L'historien reste, quant à lui, au bord de la tombe. Notre enquête de terrain n'est pas allée non plus jusqu'à l'observation, notamment participante, que pratiquent les anthropologues et les sociologues pour les métiers de la mort ${ }^{32}$, manque compensé partiellement par quelques entretiens ${ }^{33}$. L'historien dispose donc d'une grande diversité de sources et d'outils méthodologiques enrichis par l'archéologie, mais aussi par l'anthropologie, la sociologie ou l'histoire de l'art.

28. Le premier était à l'origine spécialiste d'archéologie grecque, avant de créer une chaire d'archéologie moderne; le second d'archéologie moderne et contemporaine.

29. Revue d'archéologie moderne et d'archéologie générale. La quasi-totalité des articles portant sur l' "archéologie de la mort» ont été écrits par Pierre-Yves Balut.

30. Par exemple, Guénola Groud, responsable depuis 2007 de la cellule patrimoine du Service des cimetières de la Ville de Paris. Sur sa démarche, voir : "Un inventaire du patrimoine funéraire parisien. Sources, méthode et critères de patrimonialisation ", in Bruno Bertherat (dir.), Les Sources..., op. cit., p. 267-281.

31. Par exemple, Gian Marco Vidor, «Écrits pour les morts. Les graffitis et les messages sur papier dans les cimetières : fragilité d'une source historique (Espagne, France, Italie) ", in Bruno Bertherat (dir.), Les Sources..., op. cit., p. 345-361.

32. Par exemple, Julien Bernard, Croquemort. Une anthropologie des émotions, Paris, éd. Métailié, 2009, qui nous a communiqué un extrait de son journal de terrain.

33. Avec Sylvie Lefebvre, conservatrice du cimetière communal de Bagneux, et trois de ses collaborateurs, fossoyeurs au cimetière : Léo Baret, Nasser Hebbabla, Fabien Pascaud. Avec Philippe Vidal et Nicolas Weydert, archéologues à l'Inrap. 
C'est par l'imagination scientifique, qui fait le lien entre les différentes approches, qu'il pourra alors tenter de comprendre la tombe de Jeanne, dans l'espace, visible et invisible, et dans le temps, court et long ${ }^{34}$.

\section{UNE «CAPSULE DE TEMPS $»^{35}$}

La tombe de Jeanne est d'abord l'expression matérielle d'un temps figé, celui de son souvenir. La concession perpétuelle et le tombeau, permis par le décret de prairial, deviennent la concrétisation ultime de ce lien instauré par les vivants avec cette petite morte. Il faut alors en retrouver la logique et les signes, de la surface au monde d'en bas où repose Jeanne.

Inhumée le 12 mars, Jeanne a connu deux sépultures : la première en terrain commun ou gratuit, puis une concession perpétuelle, située l'une et l'autre dans la partie bénite par l'Église, accessible aux seuls baptisés ${ }^{36}$. Ce changement de lieu et de statut correspond aux deux extrêmes de la hiérarchie sociale des tombes à l'échelle de ce petit cimetière communal, alors qu'à Paris elle s'opère aussi entre les cimetières. Les nouveaux cimetières parisiens extra muros créés à partir du Second Empire sont en effet affectés aux inhumations à durée limitée. À la mort de Jeanne, seuls les cimetières parisiens de Saint-Ouen et d'Ivry sont en activité. On ignore l'emplacement exact de la première sépulture de Jeanne. Elle est située à l'intérieur d'un des six carrés que comporte le cimetière (trois de chaque côté de l'allée centrale), les bords étant réservé aux concessions, et dans l'espace destiné aux enfants, à proximité de celle de son frère Arthur-Victor, mort en 1869 à l'âge de quelques semaines (à l'époque où les parents de Jeanne habitaient et travaillaient à Bagneux $)^{37}$. Le regroupement des tombes d'enfants est courant dans une période où leur mortalité est encore importante, surtout dans les classes populaires d'où provient Jeanne ${ }^{38}$ et correspond aux recommandations de

34. Nous tenons à remercier toutes les personnes citées pour leur aide dans les notes de bas de page précédentes et suivantes, ainsi que Régis Bertrand, Françoise Bonnecaze, Antoine Bourroux, Guy Flucher, Guénola Groud, Antoinette Guise Castelnuovo, Hervé Guy, Dominique Jacquier, Franck Javary, Tom Jolit, Henry La Fontaine, Christine Lallemand, Dominique Lecomte, Julien Le Magueresse, Clément Lorillec, Arnaud Maillet, Valérie Maillet, Amandine Malivin, Nathalie Minart, Françoise Monfrin, Juliette Nunez, Ana Maria Olmedo, Philippe Ploix, Anne Richier, Gisèle Rousseau, Monique Sicaud-Bioret, Isabelle Tarisca, Vincent Thauziès, Françoise Thébaud, Gian Marco Vidor, Gabrielle Vitali.

35. Nous détournons ici le sens du concept développé notamment par William Jarvis (Time Capsules. A Cultural History, 2002). La tombe instaure un continuum en partie visible entre passé, présent et avenir.

36. Jusqu'à la loi du 14 novembre 1881 mettant fin au caractère confessionnel des cimetières.

37. ACB, 1E16, 1866-1873, Acte de décès du 20 mars 1869 et 271W38, Concessions de cimetière, $1865-1874, \mathrm{n}^{\circ} 141,20-21$ mars 1869 . Le couple aurait eu un autre enfant avant Jeanne, mais nous ne savons pas encore quand il est mort ni où il est enterré.

38. Le grand-père maternel de Jeanne est charron. Victor Moyaux a exercé plusieurs métiers : ouvrier charron chez son beau-père, puis ouvrier chapelier, comme son épouse, et employé dans une maison de crédit à Paris. La grand-mère maternelle de Jeanne, couturière, morte en 1874 est également enterrée en terrain commun dans le cimetière communal. 
l'Église pour les anciens cimetières paroissiaux ${ }^{39}$. Il permet un gain d'espace, car ces tombes sont plus petites que celles des adultes. La durée d'occupation d'une tombe en terrain commun est théoriquement limitée à cinq ans, durée estimée par le législateur pour la décomposition du corps (et qui est jugée insuffisante au cimetière communal de Bagneux), afin de permettre une rotation des sépultures. On remarquera que la tombe du frère de Jeanne est encore en place huit ans après son inhumation.

Le 12 juin $^{40}$, le corps de Jeanne est transféré dans une concession perpétuelle d'adulte, jouxtant le mur gauche du cimetière (fig.2), concession couronnée d'un tombeau en septembre. Le transfert et le tombeau sont ici exceptionnels, car c'est une victime innocente que la commune a voulu honorer. Cet hommage avait été annoncé par les funérailles de mars qui avaient été prises en charge par la Préfecture de police, mais aussi sans doute par les institutions ou associations religieuses et communales ${ }^{41}$. Quant à la concession et au tombeau, ils sont offerts par la commune à la suite d'une souscription ouverte par le maire Philippe Leviaux (en fonction depuis 1870) qui apparaît comme le concessionnaire, et relayée par la presse. L'ensemble est inscrit dans deux registres contenant environ 200 noms de souscripteurs. La quasi-totalité semble demeurer à Bagneux, ce qui signifierait que $13 \%$ environ des habitants ont versé de l'argent. Il faudrait rechercher la logique de ces dons en examinant la liste des souscripteurs et leurs liens possibles avec Jeanne. Quoi qu'il en soit, c'est un témoignage très fort de l'engagement de la population en faveur de la mémoire de la petite fille. Bien qu'elle ait réclamé le corps ${ }^{42}$, la famille est ici en retrait, parce qu'elle n'est plus légitime : le père est le meurtrier et la mère est discréditée, en particulier auprès de la population balnéolaise, accusée d'avoir poussé par son infidélité son mari au crime $^{43}$. C'est dans la commune de son grand-père maternel, et non à Paris, que la petite est enterrée (le maire a dû donner son accord) et il est apparemment le seul membre de la famille à être autorisé à suivre les funérailles. La mère aurait été néanmoins présente lors de la seconde inhumation ${ }^{44}$.

39. Régis Bertrand, "Sépultures et tombeaux d'enfants (France, $\mathrm{XVIII}^{\mathrm{e}}$-XIX ${ }^{\mathrm{e}}$ siècle). Quelques remarques", in Charles Zaremba (dir.), La Mort de l'enfant. Approches historiques et littéraires, Aix-enProvence, Publications de l'Université de Provence, 2011, p. 37. du 19.

40. Date figurant sur le registre d'inhumation. Le Petit Journal (20 juin 1877, p. 3) évoque celle

41. Le fourgon mortuaire est celui de la Morgue qui dépend de la Préfecture de police; son cercueil est porté par les jeunes filles de l'institution des sœurs de Saint-Vincent-de-Paul; il est suivi par l'instituteur et les élèves de l'école primaire de garçons; la fanfare de Bagneux est aussi présente, etc (APP, BA81, Dossier Affaire Moyaux, Rapport du 12 mars 1877, fol. 170; Le Petit Parisien, 14 mars 1877 , p. 2-3, Le Figaro, 13 mars 1877, p. 2 par exemple).

42. APP, Registre de la Morgue, $1877, \mathrm{n}^{\circ} 45,5$ février.

43. APP, BA81, Dossier Affaire Moyaux, Rapport du 12 mars 1877, fol. 170 et Le Figaro, 13 mars 1877, p. 2. C'est la raison pour laquelle Victor Moyaux n'est pas condamné à mort, mais aux travaux forcés à perpétuité à l'issue de son procès en mai. Il effectue sa peine en Nouvelle-Calédonie jusqu’à sa mort en 1908 : Archives nationales d'Outre-Mer (ANOM), H 729 MOYAUXVICT.

44. Selon Le Petit Journal, 20 juin 1877, p. 3. 
Fig. 3 : La tombe de Jeanne Moyaux au cimetière municipal de Bagneux (photographie de Françoise Monfrin, 4 juillet 2018). Crédits photographiques: Françoise Monfrin.

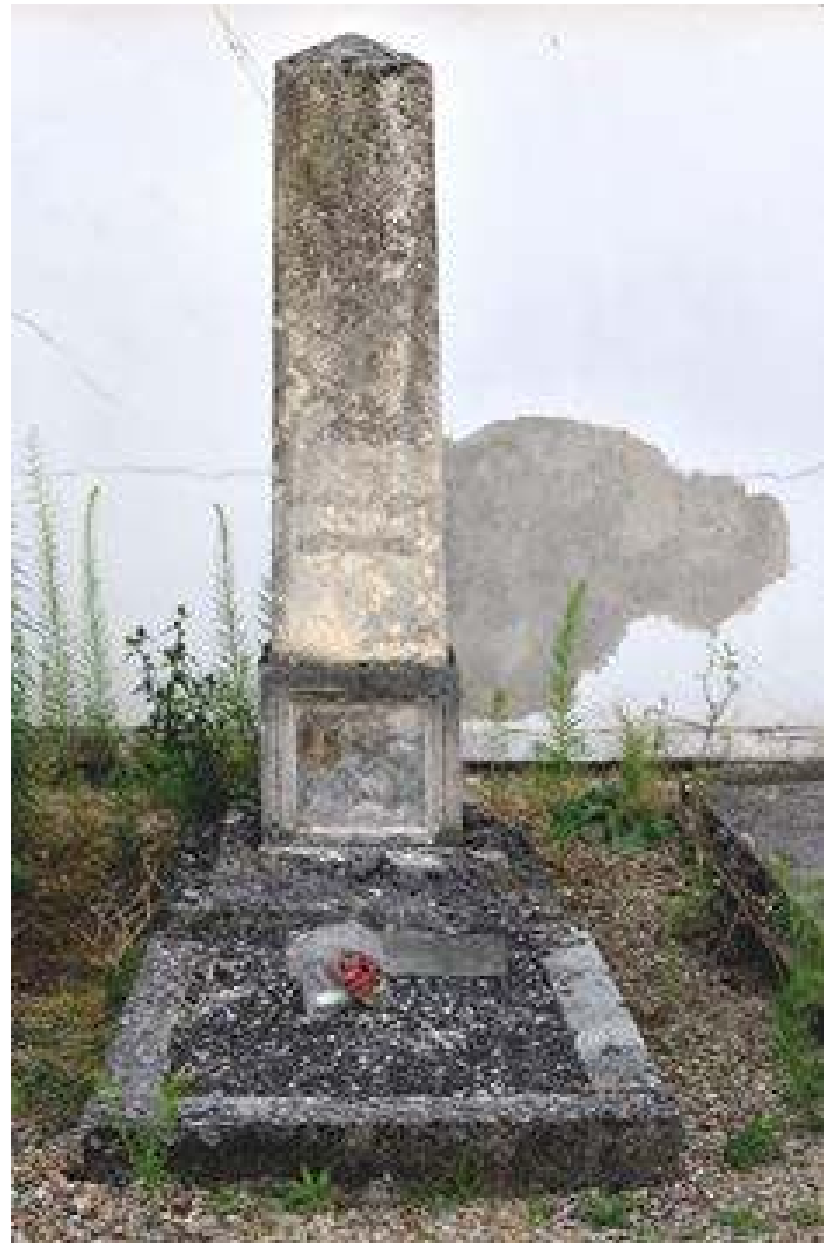

Des funérailles au tombeau, l'hommage à la petite fille est avant tout celui rendu par la communauté. Elle est distinguée par-delà la mort par la monumentalité de sa tombe qui s'offre aux regards de tous dans l'espace du cimetière.

Monument pérenne autorisé par le décret de prairial comme la concession perpétuelle, auquel il est associé, le tombeau apparaît comme le signe extérieur le plus fort du culte des morts. Son prix peu élevé (137,60 francs) explique sa modestie, qui contraste avec les monuments plus élaborés des enfants de la haute société dans d'autres cimetières. Une modestie assumée dans l'intitulé de la souscription et qui explique aussi sans doute le choix de l'inhumation en pleine terre et l'absence de semelle ${ }^{45}$. Le monument, tel qu'il nous apparaît aujourd'hui (fig. 3), est constitué d'une stèle de 2,10 m

45. La semelle est la structure posée sur le sol qui délimite le pourtour de la concession et sur laquelle est posé le monument funéraire auquel elle confere assise et stabilité. 
de hauteur en forme de pilier quadrangulaire, dans une pierre calcaire claire qui provient probablement de carrières voisines, reposant sur une plaque de ciment et surplombant un soubassement rectangulaire en pierre avec une chape en ciment recouverte de graviers. La forme, qui n'est pas spécifique aux tombeaux d'enfants, rappelle les monuments inspirés de l'Antiquité, apparus notamment au Père-Lachaise ${ }^{46}$, et qu'on retrouve au cimetière de Bagneux, mais aussi les monuments commémoratifs érigés à la même époque. Le tombeau de Jeanne s'inscrit dans le paysage banal d'un cimetière d'une petite commune de la banlieue parisienne, peuplé de dalles, de sarcophages, de stèles et parfois de tombeaux plus élaborés ou plus simplement, dans le terrain commun, de ces rangées de croix pour les adultes et de petites croix blanches pour les enfants que Zola décrivait pour le cimetière parisien de Saint-Ouen ${ }^{47}$. On notera que Marthe Erbelding, la jeune victime de la célèbre affaire Soleilland (1907) inhumée au cimetière parisien de Pantin, ne dispose que d'une concession temporaire et rien n'indique l'érection d'un tombeau ${ }^{48}$.

Montrer l'emplacement du mort, c'est aussi commémorer son nom, parfois faire son éloge et en regretter l'absence. Le tombeau appelle l'épitaphe. Celui de Jeanne ne déroge pas à la coutume. Grâce aux deux clichés de la collection Rousseau, on peut déchiffrer les inscriptions creusées en lettres capitales peintes en noir dans la pierre de la stèle : «À la mémoire/ de MarieJeanne/ Moyaux / décédée / le 5 février 1877 / Les habitants de Bagneux». Ne sont pas évoquées les conditions de la mort ni son statut d'enfant. On trouve également sur la base cubique de la stèle un cadre en verre. Il protégeait peutêtre une photographie de la petite fille prise de son vivant ${ }^{49}$. Mais l'hypothèse devrait être étayée par une comparaison avec des monuments équivalents dans ce cimetière ou ailleurs. Cet hommage de la communauté fait écho à celui, familial, rendu quelques années plus tôt au frère de Jeanne, tel qu'il est retranscrit par la presse : «Ici repose le corps de Arthur-Victor Moyaux décédé le 20 mars, à l'âge de 8 semaines, regretté de ses père et mère et toute sa famille D. P. [De profundis] ${ }^{50}$ ". Ce type d'inscriptions montre que la mort de l'enfant devient «de plus en plus publiquement un drame», y compris dans les classes populaires, à une époque caractérisé par la baisse de la natalité et la promotion croissante de l'enfant au sein de la famille et de la sociétés1. Jeanne aurait pu bénéficier d'une telle épitaphe, si elle était morte dans des circons-

46. César Daly, Architecture funéraire contemporaine. [...], Paris, Ducher, 1871.

47. Émile Zola, L'Euvre, Paris, Gallimard, 1983 [1886], p. 405.

48. Renseignements sur la concession obtenus auprès de la conservation de ce cimetière. Et pourtant Marthe a bénéficié de funérailles grandioses à l'aune de l'éclat médiatique de l'affaire (Le Petit Parisien, 15 février 1907, p. 1-2). Voir Jean-Marc Berlière, Le Crime de Soleilland (1907). Les journalistes et l'assassin, Paris, Tallandier, 2003.

49. APP, YB 9.

50. Le Figaro, 13 mars 1877, p. 2.

51. Nous empruntons cette formule à Régis Bertrand, extraite d'une communication non encore parue. Voir aussi Michel Vovelle, La Mort et l'Occident..., op. cit., p. 615-620; Marie-France Morel, "Images du petit enfant mort dans l'histoire», L'Esprit du temps, n 119, 2001/1, p. 17-38; Régis 
tances similaires. On remarquera pour finir l'absence de référence religieuse sur le tombeau de Jeanne, qui contraste avec la croix blanche surmontant la tombe du frère ${ }^{52}$. La dimension commémorative l'emporte.

$\mathrm{Si}$ le tombeau est sémaphore, il n'est pas cénotaphe : il est la partie émergée de la tombe et perpétue le souvenir de l'être aimé qui lui n'est plus visible, mais qui demeure. Reste alors à reconstituer le «salon noir» de Jeanne ${ }^{53}$.

Le cercueil qu'on descend dans la fosse, le bruit de la terre qu'on jette, les gestes des fossoyeurs, tout renvoie à des pratiques qui semblent immémoriales. L'âme de Jeanne est sauvée, puisqu'elle est morte en âge d'innocence; le corps retourne à la poussière jusqu'au Jugement dernier : «Ne regrettez pas l'enfant [...], elle est au ciel», aurait dit le prêtre de la paroisse lors de la cérémonie à l'église ${ }^{54}$. Si le cimetière de prairial reste un espace de dissolution des corps, au nom de l'hygiène, il doit néanmoins composer avec le culte des morts. Comment dans ces conditions accepter la dissolution de l'être aimé? La présence du signe à la surface ne fait pas disparaître ce qu'il cache : le cimetière et l'objet funéraire sont l'expression d'une "société de conservation", qui nie en quelque sorte le cadavre ${ }^{55}$. Cette injonction a des incidences sur le corps, dès avant l'inhumation. La vogue du bijou-souvenir, de la photographie, voire du masque mortuaire, l'essor de l'embaumement, voulu comme définitif et dans le respect de l'intégrité physique, sont autant de pratiques, associées aux classes supérieures, visant à assurer une survie matérielle du $\operatorname{corps}^{56}$. Jeanne présente un cas particulier, car elle échappe à sa famille dès sa mort. C'est ainsi qu’à la Morgue le corps est photographié assis et habillé57, puis exposé dans cet état pour être plus facilement identifié. Il est ensuite autopsié le 6 février, puis embaumé le 10 par le conservateur des pièces anatomiques de la Faculté de médecine de Paris afin qu'il puisse être présenté à son père qui n'est arrêté que le 9 mars, ce qui explique le long délai

Bertrand, «Sépultures et tombeaux d'enfants (France, $\mathrm{XVIII}^{\mathrm{e}}$-XIX ${ }^{\mathrm{e}}$ siècle)... », art. cit.; Catherine Rollet, Les Enfants au XIX' siècle, Paris, Hachette Littératures, 2001.

52. Nous ne savons pas l'orientation politique du maire. Un indice : il est élu pour la première fois le 6 septembre 1870, juste après la chute de l'Empire et la proclamation de la République.

53. Nous détournons le sens du titre de l'émission de Vincent Charpentier sur France Culture consacrée à l'archéologie (le nom a changé récemment : Carbone 14).

54. Selon Le Figaro (13 mars 1877, p. 2), qui ajoute : «demandant pardon à Dieu pour les entrailles qui l'ont portée et pour celui qui l'a engendrée" (voir aussi APP, BA81, Dossier Affaire Moyaux, Rapport du 12 mars 1877, fol. 170), formulation qui assimile l'enfant à un ange intercesseur. Sur ce sujet, voir en particulier Régis Bertrand, "Les enfants "qui remplissent le ciel". Obsèques et sépultures des enfants en Provence aux XVII ${ }^{-}$-XVIII ${ }^{e}$ siècles", in Mort et Mémoire..., op. cit., p. 101-113 et "Sépultures et tombeaux d'enfants (France, XviII ${ }^{e}$-XIX" siècle)... », art. cit., p. 40-44.

55. Jean-Didier Urbain, La Société de Conservation. Étude sémiologique des cimetières d'Occident, Paris, Payot, 1978.

56. Voir Philippe Ariès, L'Homme devant la mort, op. cit., p. 452-455; Emmanuelle Héran (dir.), Le Dernier Portrait. Musée d'Orsay, Paris, 5 mars-26 mai 2002, Paris, Éditions de la Réunion des musées nationaux, 2002; Emmanuel Fureix, La France des larmes. Deuils politiques à l'àge romantique (1814-1840), Seyssel, Champ Vallon, 2009, p. 55-59; Anne Carol, L'Embaumement, une passion romantique, Seyssel, Champ Vallon, 2015.

57. APP, YB 9. 
entre sa mort et son identification et son inhumation. L'ambiguïté avec les usages funéraires est ici frappante.

La mise en bière de Jeanne, effectuée également à la Morgue, montre la même ambiguïté. Cette opération, à la fois hygiéniste et funéraire, marque la disparition définitive du corps de l'espace visible. Les cercueils de verre font partie de la fantasmagorie ${ }^{58}$ ou de l'exception, comme celui qui contient la petite Rosalia Lombardo, morte en 1920, dont le corps embaumé est conservé dans les Catacombes des Capucines à Palerme. Il y a pourtant une mise en scène qui se déroule dans l'intimité et n'est décrite pour Jeanne dans aucune des sources consultées. La Morgue offre il est vrai un théâtre particulier où l'administratif, voire le judiciaire, l'emportent a priori sur le funéraire. Le cercueil en sapin de Jeanne est celui des pauvres et des corps non réclamés ou non identifiés. L'enfant est-elle habillée, comme il est désormais d'usage pour tous? Et avec les vêtements qu'elle portait au moment de sa mort? Des objets l'accompagnent-elle? Une couronne de fleurs déposée sur la tête, pratique préconisée par le Rituel romain de 1614 pour les enfants baptisés morts avant l'âge de raison ${ }^{59}$ ? Des objets religieux (médaillon, crucifix)? Un jouet? On pense ici à une poupée. Inscrite dans la longue durée, la pratique des offrandes dépend de l'âge et du genre du défunt, mais aussi des circonstances ${ }^{60}$. Quelle part y a prise la famille? Le grand-père s'est peut-être rendu à la Morgue, après l'identification de Jeanne, pour demander son inhumation. La mère et sa sœur étaient peut-être dans le fourgon qui accompagnait le corps de la Morgue à Bagneux (et donc présentes lors de la mise en bière) ${ }^{61}$. Le personnel a-t-il pratiqué envers le corps des gestes funéraires? Et quel rôle a joué la communauté balnéolaise? D'une certaine manière, le cercueil est habité par le souvenir des vivants, qui accompagne l'être cher au-delà de la mort en subvertissant les frontières.

La tombe de Jeanne matérialise ainsi sa mémoire portée par les vivants, bien au-delà de sa dimension hygiénique. Mais la mémoire est comme les vivants, mortelle. Si la tombe tente de figer le temps, elle n'en évite pas l'usure.

58. On renvoie au mémoire de l'HDR de Stéphanie Sauget, soutenue le 5 mai 2018 (Le Cercueil de verre ou l'imaginaire de la "dernière demeure») et plus largement à ses recherches sur le cercueil.

59. Régis Bertrand, "Des fleurs pour les morts: aux origines des offrandes florales contemporaines", in Mort et Mémoire, op. cit., p. 140.

60. La pratique du dépôt d'objets religieux et de jouets est notamment attestée par Arnold Van Gennep (Le Folklore français, vol. 1, Du berceau à la tombe..., op. cit., p. 615). On la retrouve sur les photographies mortuaires. Ces constatations rejoignent les observations de Julien Bernard dans son carnet d'enquête.

61. Le Petit Journal, 11 mars 1877, p. 2; Le Temps, 14 mars 1887, p. 2. La mise en bière de Marthe Erbelding à la Morgue en présence de sa famille est en revanche largement décrite (Le Petit Parisien, 15 février 1907, p. 1). 


\section{L'USURE DU TEMPS}

La tombe de Jeanne est un maillon essentiel de son souvenir. Elle suppose des usages qui prennent fin quand le souvenir s'estompe. Il faut alors voir quand et comment se noue, puis se dénoue la chaîne des temps. Quelle est la litanie des hommages à la tombe? Quel est son destin mémoriel et matériel?

Tous les regrets sont d'abord éternels. Les hommages à Jeanne commencent sans doute dès sa mort, mais c'est au moment des funérailles, qui attirent plusieurs milliers de personnes des environs et jusqu'à Paris, qu'ils deviennent vraiment visibles dans les sources policières et médiatiques. Les offrandes de fleurs, des funérailles à la tombe, incarnent peut-être le mieux avec le tombeau la dimension laïque du culte des morts contemporains. Elles se sont développées à partir de la Révolution française en reprenant des usages hérités de l'Antiquitée ${ }^{2}$. À l'époque de Jeanne, la pratique est généralisée. La presse évoque des bouquets et des couronnes de violettes et d'immortelles et des couronnes de perles blanches, offertes notamment par les sœurs de SaintVincent-de-Paul, mais aussi par des anonymes. On les retrouve sur le cercueil lorsqu'il est exposé devant la maison du grand-père et bien sûr sur la tombe, après la descente du cercueil. Violettes et immortelles sont associées au funéraire, tandis que la blancheur de la couronne de perles renvoie à la pureté et à l'innocence de l'enfance. D'autres signes montrent l'importance du deuil : l'affliction, dont témoignent entre autres les mots du maire sur la tombe, les pleurs de ses petites camarades, des évanouissements de femmes (entre topos et réalité), les vêtements de deuil eux-mêmes, les musiques funèbres jouées de l'église jusqu'au bord de la tombe. L'atmosphère est aussi marquée par l'excitation de la curiosité, voire du voyeurisme, avivée par le fait-divers, qui explique le bruit et les mouvements de foule. Que se passe-t-il quand s'estompe le fait-divers?

Au temps court des funérailles succède le temps long qui transforme le deuil en souvenir et dont les échos se retrouvent dans les usages de la tombe de Jeanne. Au moment de ses funérailles, un journal observe que la tombe de son frère est "très bien entretenue ${ }^{63}$ ", ce qui est conforme au culte familial des morts et explique peut-être en partie qu'elle n'ait pas été encore reprise. Quant à la tombe de Jeanne, on peut supposer que ce sont les habitants de Bagneux qui ont pris le relais, à défaut de la famille $e^{64}$, en continuant à se rendre sur la tombe pour y déposer des fleurs ou d'autres objets funéraires à certaines dates symboliques, comme l'anniversaire de la mort et surtout la

62. Régis Bertrand, «Des fleurs pour les morts...», art. cit.

63. Le Figaro, 13 mars 1877 , p. 2.

64. Le grand-père n'apparait plus dans la liste des habitants de la commune dans les recensements et n'est ni décédé ni inhumé dans celle-ci. Au moment de l'affaire, la mère de Jeanne vivait chez son amant à Paris. On perd ensuite sa trace. On sait seulement qu'elle se trouvait en Belgique au moment de la mort de son mari (ANOM, H 729 MOYAUXVICT). 
Toussaint et la fête des morts ${ }^{65}$. On peut penser également que certains des visiteurs réguliers de Jeanne font partie de la liste des souscripteurs, tels le maire ou la veuve Marchais, la nourrice balnéolaise de Jeanne, qui semblait très attachée à elle ${ }^{66}$. Cette pratique de la visite à la tombe n'est pas attestée par la presse, alors qu'elle le sera plus tard pour la tombe de Marthe Erbelding, probablement en raison de la faible notoriété du cimetière communal de Bagneux ${ }^{67}$. Pour la même raison, il est douteux que la tombe de Jeanne ait suscité ce tourisme funéraire qui caractérise les grands cimetières parisiens ${ }^{68}$. Et nous n'avons pas de trace d'un quelconque pèlerinage. Sur la longue durée, les hommages se sont sans doute limités à la communauté balnéolaise et la tombe de Jeanne ressemblait peut-être dans les premières années à celle de la "petite martyre de l'autoroute», située dans le cimetière de la commune de Suèvres (Loir-et-Cher), où son corps sans nom avait été découvert en $1987^{69}$. La comparaison seule permet ici de pallier le silence des sources.

La seule trace qui demeure aujourd'hui sur la tombe de Jeanne est une plaque ornée d'une fleur rose en céramique et dépourvue d'inscription, mais lui était-elle destinée à l'origine ${ }^{70}$ ? Il faut alors inverser la perspective et analyser le processus d'oubli et ses conséquences.

Si l'abandon est l'œuvre du temps, il faut en comprendre le rythme et les raisons en distinguant bien la déritualisation que le rite lui-même implique, comme l'abandon progressif des vêtements de deuil, et le délitement du souvenir qui met fin à tout. Ce dernier est lié à la disparition des contemporains de Jeanne ${ }^{71}$. Le maire qui était resté en fonction jusqu'en 1881 (après une brève interruption pendant l'année 1879) meurt en 1885 à l'âge de 80 ans. Son tombeau est situé près de l'allée centrale, dans le prolongement de celui de Jeanne, à quelques mètres à peine. Le souvenir a sans doute été porté plus longtemps par les petites camarades de Jeanne et peut-être audelà même d'une génération. Pour les nouvelles générations cependant, la petite fille devient peu à peu une inconnue. Dans la presse, la mémoire du crime et du meurtrier l'emporte en général très tôt sur celle de la tombe. Les circonstances de la mort ont pu jouer un rôle d'accélérateur, comme le montre l'ambivalence du bref discours du maire sur le bord de la tombe : «Pauvre petite victime, repose en paix avec les regrets de tous les habitants de

65. Jean-Hugues Déchaux, Le Souvenir des morts. Essai sur le lien de filiation, Paris, PUF, 1997; Jacqueline Lalouette, Jours de fête. Fêtes légales et jours fériés dans la France contemporaine, Paris, Tallandier, 2010, p. 84-95.

66. Comme cela a été dit lors du procès. Voir par exemple Le Petit Journal, 16 mai 1877, p. 3.

67. Étude comparée dans Le Petit Parisien à partir de Gallica. Occurrences concernant la tombe de Marthe : chaque 2 novembre des années 1908 à 1911.

68. Madeleine Lassère, "Le XIX siècle et l'invention du tourisme funéraire", Revue d'histoire moderne et contemporaine, $\mathrm{n}^{\circ}$ 44-4, octobre-décembre 1997, p. 601-616.

69. Une des expressions employées par les médias pour désigner le cadavre de cette petite fille découvert sur le bord de l'autoroute A10.

70. L'objet semble plus récent que la tombe (début du $\mathrm{xx}^{\mathrm{e}}$ siècle?). Les vols et les déplacements d'offrandes funéraires sont une réalité de la vie des cimetières.

71. Maurice Halbwachs, Les Cadres sociaux de la mémoire, Paris, Albin Michel, 1994 [1925], p. 166-167. La communauté balnéolaise est ici un substitut de la famille. 
Bagneux, ce pays si tranquille d'ordinaire, et à qui ta mort a conquis une si triste célébrité $!^{72} »$. La mort de Jeanne est donc une tache dans la réputation du village. Rendre hommage à la victime, c'est d'une certaine manière rappeler le meurtre, tout en le réparant symboliquement. Jeanne renvoie à un souvenir douloureux pour la communauté, d'autant plus que le meurtrier, s'il n'était pas originaire de la commune, y avait vécu et travaillé pendant deux ans, de 1867 à 1869, et s'y était marié avec une habitante ${ }^{73}$. Bagneux n'est pas Paris : l'onde de choc du crime y est probablement plus forte.

Aujourd'hui le souvenir de Jeanne est tombé dans l'oubli, un oubli que l'on retrouve, volontaire ou non, depuis longtemps dans les ouvrages sur Bagneux $^{74}$. L'éloignement progressif de l'événement et les mutations profondes de la commune ont fait le reste. Un siècle après la mort de Jeanne, l'association Les Amis de Bagneux publie dans son bulletin le premier récit historique de l'affaire. L'auteur anonyme, qui est sans doute René Rousseau, indique : «Des personnes âgées se souviennent encore du puits à Moyaux ${ }^{75}$ ». Ce sont les derniers échos dont nous ayons connaissance et l'histoire de la tombe elle-même était inconnue du personnel actuel du cimetière. Philippe Landru, auteur d'un site de référence sur l'histoire des cimetières français, ne l'évoque pas non plus ${ }^{76}$. En revanche, Bagneux a ses héros, dont elle entretient la mémoire, comme le commandant de Dampierre, mort dans des combats contre les Prussiens en octobre 1870 qui ont marqué l'histoire de la commune. La place de l'ancien cimetière porte son nom et un monument aux morts dit du commandant de Dampierre est érigé sur une place de Bagneux, puis transféré au cimetière au moment de son agrandissement dans la partie ancienne où il demeure aujourd'hui, à quelques dizaines de mètres de la tombe de Jeanne. Si l'affaire Moyaux est totalement oubliée, un autre fait-divers a profondément marqué l'histoire récente de Bagneux et présente des similitudes troublantes, malgré des circonstances différentes: l'affaire Ilan Halimi, dite aussi du "Gang des barbares», en 2006. Ce crime crapuleux et antisémite a suscité une profonde émotion à Bagneux et ses édiles et ses habitants sont partagés entre la volonté de rendre hommage à la victime et celle d'oublier l'horreur de l'acte ${ }^{77}$, un peu comme pour Jeanne.

Le destin mémoriel de Jeanne ne lui est pas propre. Toutes et tous sont concernés à plus ou moins brève échéance par la mort du souvenir, à l'excep-

72. Le Figaro, 13 mars 1877 , p. 2. La version du rapport de police est légèrement différente sur la forme, pas sur le fond (APP, BA81, Dossier Affaire Moyaux, Rapport du 12 mars 1877, fol. 170).

73. Victor Moyaux est originaire de l'Indre. Son épouse est née à Arcueil, commune limitrophe de Bagneux.

74. Eugène Toulouze, Histoire d'un village ignoré (Balnéolum), Paris, Paul Schmidt, 1898; État des communes à la fin du XIX siècle. Bagneux..., op. cit.; Madeleine Leveau-Fernandez et Antoine Bertoncini, Bagneux. Des origines à nos jours, Bagneux, Ville de Bagneux, 1986.

75. "Il y a cent ans l'affaire Moyaux bouleversait Bagneux", Bulletin de l'association Les Amis de Bagneux, n 5,1977 , p. 13.

76. https://www.landrucimetieres.fr/spip/spip.php?article2916 (page consultée le 24 juin 2018).

77. Ilan Halimi a été inhumé à Jérusalem. Une plaque en son hommage a été installée dans un parc à Bagneux et un arbre a été planté. 
tion des grands hommes au sens large qui bénéficient d'un répit lié à leur statut. Restent alors les vestiges de son tombeau.

Le tombeau de Jeanne, l'un des plus anciens du cimetière, n'est plus entretenu depuis longtemps. La pierre noircit et les lettres s'effacent. Ces dégradations sont dues aux intempéries et aux champignons qui rongent le calcaire, plus poreux que le granit. On en retrouve les effets sur le cadre à la base de la stèle dont la plaque de verre extérieure est tombée. Le vandalisme a-t-il sa part? L'article de 1977 affirme en effet que «jadis, une main inconnue a gratté le nom de Marie-Jeanne Moyaux ». Cette interprétation n'est étayée par aucune source et, pour le personnel du cimetière, la dégradation est naturelle. La tombe a en revanche subi des interventions : le soubassement a été réparé il y a longtemps, et la tombe est pourvue depuis le début des années 2000 au moins d'une nouvelle chape de ciment recouverte de graviers, toujours en place aujourd'hui ${ }^{78}$. On ignore qui a pris la décision de ces travaux. Enfin, la tombe commence à être cernée de plantes sauvages ${ }^{79}$. La dégradation des concessions perpétuelles due à leur abandon est un phénomène observé depuis le milieu du XIx ${ }^{e}$ siècle au Père-Lachaise. La loi du 3 janvier 1924 et le décret du 25 avril 1924 autorisent la reprise des perpétuelles abandonnées et la création d'un ossuaire spécifique; celle du 19 décembre 2008 permet la crémation des restes exhumés ${ }^{80}$. Cette évolution importante du droit funéraire permet de lutter contre le manque de place dans les cimetières urbains. Si la tombe de Jeanne n'a pas encore été reprise, c'est parce que le cimetière de Bagneux ne manque pas de place et aussi parce qu'elle n'est pas en ruine et ne pose aucun problème de sécuritét ${ }^{81}$.

Que reste-t-il du salon noir de la petite fille aujourd'hui? Pour l'imaginer, l'historien peut s'appuyer sur l'expérience de l'archéologue et du fossoyeur dégageant la fosse. La terre d'abord est omniprésente par sa masse, et ce n'est qu’au fur et à mesure qu'apparaîtront les vestiges : ceux du cercueil, fragile et disloqué, des vêtements et des objets, dont il reste parfois des indices indirects $^{82}$, et ceux enfin du corps dont la tête ou le crâne se voit généralement en premier, parce qu'il s'agit du relief le plus élevé d'un corps allongé8 ${ }^{83}$. Plusieurs

78. Ce que montre la comparaison entre les photos de René Rousseau et celles effectuées en décembre 2001 par le prédécesseur de l'actuelle conservatrice du cimetière (celui-ci avait fait des photos de la tombe voisine très dégradée dans l'éventualité de sa reprise qui n’a pas eu lieu).

79. Dont la pousse est favorisée par l'arrêt des traitements phytosanitaires en 2016.

80. «[...] en l'absence d'opposition connue, attestée ou présumée du défunt» (article 19 de la loi et L2223-4 du Code des collectivités territoriales).

81. Quant à la tombe du grand-frère, elle a disparu depuis longtemps et ses restes ont peut-être rejoint l'ossuaire, situé sur la bordure droite du cimetière de 1867, en face de la tombe de Jeanne, et fermé aujourd'hui. La tombe de Marthe Erbelding à Pantin a été reprise à une date inconnue.

82. Par exemple, des vêtements ajustés peuvent avoir disparu mais être restituables par des effets de contrainte sur le squelette. Quant aux objets, ils sont évoqués dans les témoignages recueillis aujourd'hui auprès du personnel du cimetière de Bagneux. En revanche, les rapports de fouilles consultés ne mentionnent quasiment pas la présence de jouets et aucunement celle de poupées, qui se conservent mal probablement (le petit garçon de la tombe des Crottes était accompagné d'un objet qui ressemble à un fusil), alors que la présence de fleurs artificielles est relevée.

83. Mais le crâne peut s'affaisser, en particulier celui des immatures (les très jeunes enfants). Tout 
facteurs jouent un rôle essentiel dans le processus de dissolution. La qualité du cercueil d'abord (et la profondeur de son enfouissement), la nature du sol ensuite. La terre est un milieu organique, avec sa faune souterraine d'insectes nécrophages ${ }^{84}$. En outre, certaines terres, peu acides et humides, conservent mieux les corps. Cela pourrait être le cas du cimetière de Bagneux (une terre glaise humide), mais le sol est constitué en partie de remblais, ce qui signifie que la situation peut être différente d'une tombe à l'autre. Il y a enfin l'état du corps de Jeanne que la méthode d'embaumement utilisée a littéralement pétrifié, comme si le préparateur avait voulu le conserver indéfiniment sur le modèle des embaumements funéraires de cette époque ${ }^{85}$. Que reste-t-il alors de Jeanne? À défaut de le voir, nous ne pouvons qu'imaginer les gestes de plus en plus précautionneux de l'archéologue ou du fossoyeur faisant progressivement apparaitre ce qui fut un visage, cette momie d'enfant morte avant l'âge, et leur émotion, qui est aussi la nôtre.

"Chaque tombeau est la demeure d'un mort [...]; mais il est aussi comme une parole d'adieu adressée au mort par les survivants, c'est-à-dire une expression de leurs sentiments; et, dans cette mesure, les vivants y impriment aussi quelque chose de leur image ${ }^{86}{ }$. La tombe de Jeanne nous renvoie à ce temps où la tombe était le temple du souvenir, celui porté exceptionnellement par les habitants de Bagneux et non par la famille (même si son rôle ne doit pas être ignoré). Son monde n'est pas encore le monde d'hier, bien qu'il soit sans doute en train de connaître une nouvelle transition funéraire avec en particulier l'essor de la crémation et l'autorisation de la dispersion des cendres en pleine nature, ce qui signifie que le cimetière n'est plus l'unique destination des morts ${ }^{87}$. Placée dans la très longue durée, la tombe de Jeanne ne nous est pas encore incompréhensible : son temps est le nôtre.

\footnotetext{
dépend aussi de la position du corps dans la fosse.

84. Jean-Pierre Mégnin, La Faune des cadavres. Application de l'entomologie à la médecine légale, Paris, Masson, Gauthier-Villars et fils, 1894, p. 96-108 et p. 203-209.

85. APP, Rapport général sur les travaux du Conseil d'hygiène publique et de salubrité du département de la Seine depuis 1878 jusqu’à 1880 inclusivement, Paris, Chaix, 1884, "Rapport sur les agents de conservation des substances animales [...] par $\mathrm{M}^{\mathrm{r}}$ Alph. Devergie», p. 250-251; Bruno Bertherat, La Morgue de Paris au XIX siècle (1804-1907). Les origines de l'institut médico-légal ou les métamorphoses de la machine, Thèse d'histoire, Université Paris 1, 2002, p. 368-372. Nous devons à Nicolas Delestre les éclaircissements sur la dimension technique de cet embaumement. Pour une mise en perspective historique, outre Anne Carol (L'Embaumement..., op. cit.), voir Gian Marco Vidor, "Andro-lithe et pétrification des cadavres humains au XIX siècle», Frontières, vol. 23, n 1, 2010, p. 66-73.

86. César Daly, Architecture funéraire contemporaine..., op. cit., p. 10.

87. La loi sur la liberté des funérailles du 15 novembre 1887 et le décret du 27 avril 1889 autorisent et réglementent la crémation. Le décret du 20 août 1976 autorise la dispersion des cendres dans la nature, possibilité confirmée par la loi du 19 décembre 2008. Une récente mise au point : Arnaud Esquerre, Les Os, les cendres et l'État, Paris, Fayard, 2011. Sur cette nouvelle transition funéraire, voir Guillaume Cuchet, «La mort annoncée des baby-boomers : un possible tournant anthropologique", Argument, vol. 17, n 2, 2015, p. 14-26.
} 
Notre enquête va peut-être susciter un regain mémoriel qui repoussera son exhumation, puis sa crémation et son retour définitif dans la "sépulture du souvenir» du cimetière communal de Bagneux ${ }^{88}$. Il ne s'agissait pas de nous inscrire dans un devoir de mémoire, ni même de réparer une injustice, d'autant plus que la procédure judiciaire était arrivée à son terme et que l'identité de Jeanne était connue au moment de son inhumation. Telle n'était pas le cas de "la petite martyre de l'autoroute» qui vient d'être identifiée et dont les suspects de son meurtre ont été mis en examen. C'est la curiosité seule qui a conduit notre enquête, animée par le plaisir de l'échange avec d'autres disciplines, d'autres métiers. Si des pistes restent à explorer, le souvenir de Jeanne semble avoir laissé peu de traces, en particulier dans les sources de papier, à l'exception des vestiges de pierre du cimetière de Bagneux qui permettent d'en imaginer les manques.

Pourtant, la géographie du corps de Jeanne ne se limite pas à sa tombe. Nous savons où elle est et depuis quand; nous ne savons pas où et quand elle finit. Le temps de la mort ouvre en effet sur de vastes horizons, bien plus vastes que ceux d'une vie humaine, et aux populations immenses, celles des morts, plus nombreuses que les vivants ${ }^{89}$ qui interagissent avec elles. Comment l'historien peut-il alors saisir un tel objet? Par une réflexion sur le temps qui est le cœur même de sa pratique, celui qui tisse le destin du cadavre de Jeanne. Un temps que l'on peut décliner en trois grandes temporalités : le temps court de la procédure judiciaire et médiatique, le temps long du deuil et du souvenir et le temps infini des grandes croyances qui constituent la culture occidentale contemporaine. C'est dans cette voie que cet article incite à nous engager, dans ce pari biographique qui commence où la vie s'arrête, afin de redonner à la petite fille un peu de l'éclat des étoiles mortes.

Bruno Bertherat est maître de conférences en histoire contemporaine à l'Université d'Avignon et membre du Centre Norbert Elias (UMR 8562).

88. À condition que le corps soit réduit à l'état de squelette.

89. Hervé Guy, Agnès Jeanjean et Anne Richier, "Le cadavre en procès : une introduction", Techniques et culture, $\mathrm{n}^{\circ}$ 60, septembre 2013, p. 17-29. 